KEK-TH-711

UTHEP-433

YITP-00-51

hep-th/0009107

\title{
Free Field Approach to String Theory on $\mathrm{AdS}_{3}$
}

\author{
Kazuo Hosomichi用, \\ Yukawa Institute for Theoretical Physics \\ Kyoto University, Kyoto 606-8502, Japan \\ Kazumi Okuyamal] \\ High Energy Accelerator Research Organization (KEK) \\ Tsukuba, Ibaraki 305-0801, Japan \\ and \\ Yuji Satohf \\ Institute of Physics, University of Tsukuba \\ Tsukuba, Ibaraki 305-8571, Japan
}

\begin{abstract}
We discuss the correlation functions of the $S L(2, \mathrm{C}) / S U(2)$ WZW model, or the CFT on the Euclidean $A d S_{3}$. We argue that their calculation is reduced to that of a free theory by taking into account the renormalization and integrating out a certain zero-mode, which is an analog of the zero-mode integration in Liouville theory. Based on the resultant free field picture, we give a simple prescription for calculating the correlation functions. The known exact two- and three-point functions of generic primary fields are correctly obtained, including numerical factors. We also obtain some four-point functions of primaries by solving the Knizhnik-Zamolodchikov equation, and verify that our prescription indeed gives them.
\end{abstract}

PACS: $11.25 .-\mathrm{w}, 11.25 . \mathrm{Hf}$

keywords: $S L(2, \mathbf{C}) / S U(2)$ WZW model, free fields, KZ equation, AdS/CFT correspondence

*hosomiti@yukawa.kyoto-u.ac.jp

$\dagger$ kazumi@post.kek.jp

${ }^{\ddagger}$ ysatoh@het.ph.tsukuba.ac.jp 


\section{IntroduCtION}

The $S L(2, \mathbf{R})$ WZW model is the theory of a string propagating on $A d S_{3}$ in the presence of non-zero B-field. This theory is closely related to the physics of black holes in various dimensions, and it has also an application to the AdS/CFT correspondence [1, 2, 3]. At the same time, various conventional techniques of conformal field theory allows us to analyze the theory in great detail[四, 5, 6. Although our knowledge of thie model is far from complete due to the difficulty arising from the non-compactness of the target space, many recent works[7]- [17] have clarified some of its fundamental properties.

For actual applications, the Euclidean version of the $S L(2, \mathbf{R})$ WZW model is equally important. The Euclidean $A d S_{3}$ is given by the quotient space $H_{3}^{+}=S L(2, \mathbf{C}) / S U(2)$, and the string theory on this space is also described by a WZW-like action. Since the action reduces to a particularly simple form if we use a certain coordinate system, we can analyze the theory from the Lagrangian approach [18, 19, 15. Alternatively, we can analyze the system based on a free field realization of current algebra [20]- 27], [9, 13, 14, 17]. One can also study the theory based on the symmetry and bootstrap condition [28, 29] or by solving the Knizhnik-Zamolodchikov(KZ) equation [30]-34. These different approaches are of complementary use to one another and, in order to obtain a complete understanding of this theory, it is desirable to clarify how these approaches are related to one another.

In this paper, we discuss the correlation functions of the $\mathrm{H}_{3}^{+} \mathrm{WZW}$ model. We start from the full Lagrangian and primary fields. Then by integrating out a certain zero-mode and taking into account the renormalization, it is argued that the expression of the correlators becomes that of a free theory. Using the resultant free field picture, we calculate the correlation functions explicitly and obtain the results which are consistent with other approaches.

This paper is organized as follows. In section 2 we briefly summarize the $\mathrm{H}_{3}^{+}$WZW model. In section 3 we argue how the free field picture emerges, and give a prescription for calculating correlators. Using this prescription we calculate the two- and three-point functions of primary fields, and find that the known results are correctly obtained, including numerical factors. In section 4 we obtain some four-point functions of primary fields by solving the KZ equation. We see that it can be solved explicitly if we put a certain condition on the $S L(2, \mathbf{C})$ spins of four primaries. Rewriting the solutions in a form manifestly symmetric in four vertices, we find that they can be easily reproduced from our free field prescription. We conclude with a brief discussion in section 5 .

\section{$2 \quad H_{3}^{+}$WZW MODEL}

We begin with a brief review of the $H_{3}^{+}$WZW model. We follow the notations in 15] and some details are found there. The Euclidean $A d S_{3}$ is equivalent to the quotient space 
$H_{3}^{+} \equiv S L(2, \mathbf{C}) / S U(2)$, and the sigma model on this space with non-zero NS-NS B-field is known to be described by a WZW-like action. In a certain parameterization the worldsheet action becomes

$$
S=\frac{k}{\pi} \int d^{2} z\left[\partial \phi \bar{\partial} \phi+e^{2 \phi} \partial \bar{\gamma} \bar{\partial} \gamma\right] .
$$

As in ordinary WZW models, this theory has an affine $S L(2, \mathbf{C}) \times \overline{S L(2, \mathbf{C})}$ symmetry. However, unlike ordinary WZW theories, the left- and the right-moving currents are complex conjugate to each other. An important class of operators are the primary fields

$$
\Phi_{j}(z, x) \equiv\left(e^{\phi}|\gamma-x|^{2}+e^{-\phi}\right)^{2 j},
$$

which are characterized by the following OPEs with the $S L(2)$ currents $j^{A}(z)$ :

$$
\begin{gathered}
j^{A}(z) \Phi_{j}(w, x) \sim-\frac{D^{A} \Phi_{j}(w, x)}{z-w}, \\
D^{-}=\partial_{x}, \quad D^{3}=x \partial_{x}-j, \quad D^{+}=x^{2} \partial_{x}-2 j x,
\end{gathered}
$$

and similarly with $\bar{j}^{A}$. Since $j$ merely labels the second Casimir of $S L(2, \mathbf{C})$, there must be a relation between the primary fields of spin $j$ and $-j-1$. Classically they are related by

$$
\Phi_{j}(z, x)=\frac{2 j+1}{\pi} \int d^{2} y|x-y|^{4 j} \Phi_{-j-1}(z, y),
$$

but the coefficient in the right hand side may get quantum corrections.

The action (2.1) has a very simple form and allows us to carry out the path integration explicitly. In some early works [18, 19] the theory was analyzed in the path-integral formalism, using the action (2.1) and the $S L(2, \mathrm{C})$-invariant measure

$$
\mathcal{D} g=\mathcal{D} \phi \mathcal{D}\left(e^{\phi} \gamma\right) \mathcal{D}\left(e^{\phi} \bar{\gamma}\right) .
$$

There, it was shown that, after a suitable treatment of divergences arising from zero-mode integrals, one can obtain finite result for various correlators. Based on this formalism, a recent work [15] has given the two- and three-point functions of primary fields, which agree with the results in [28, 29].

\section{REDUCTION TO FREE-THEORY REPRESENTATION}

\subsection{General Argument}

To start our argument, we first rewrite the action (2.1) by introducing auxiliary fields $\beta$ and $\bar{\beta}$, and obtain

$$
\begin{aligned}
S & =S_{0}+S_{\mathrm{int}} \\
S_{0} & =\frac{1}{\pi} \int d^{2} z(k \partial \phi \bar{\partial} \phi-\beta \bar{\partial} \gamma-\bar{\beta} \partial \bar{\gamma}), \\
S_{\mathrm{int}} & =-\frac{1}{k \pi} \int d^{2} z \beta \bar{\beta} e^{-2 \phi} .
\end{aligned}
$$


The $S L(2)$ invariant measure is then

$$
\mathcal{D} \phi \mathcal{D}\left(e^{\phi} \gamma\right) \mathcal{D}\left(e^{\phi} \bar{\gamma}\right) \mathcal{D}\left(e^{-\phi} \beta\right) \mathcal{D}\left(e^{-\phi} \bar{\beta}\right)
$$

Using the above action and measure, correlators are defined by

$$
\langle X\rangle \equiv \int \mathcal{D} \phi \mathcal{D}\left(e^{\phi} \gamma\right) \mathcal{D}\left(e^{\phi} \bar{\gamma}\right) \mathcal{D}\left(e^{-\phi} \beta\right) \mathcal{D}\left(e^{-\phi} \bar{\beta}\right) \exp [-S] \cdot X
$$

Although the above action seems almost free, it still describes an interacting theory because of the term $S_{\mathrm{int}}$. In terms of the fields in (3.1), the affine symmetry of the original action (2.1) is translated into the symmetry under

$$
\begin{aligned}
& \delta \gamma=\epsilon(\gamma), \quad \delta \bar{\gamma}=-\frac{1}{2} \epsilon^{\prime \prime} e^{-2 \phi}, \quad \delta \phi=-\frac{1}{2} \epsilon^{\prime}, \\
& \delta \beta=-\epsilon^{\prime} \beta+\frac{k}{2} e^{2 \phi} \partial\left(e^{-2 \phi} \epsilon^{\prime \prime}\right), \quad \delta \bar{\beta}=0 .
\end{aligned}
$$

Here, $\epsilon(\gamma)=\epsilon_{-}(z)+\epsilon_{3}(z) \gamma+\epsilon_{+}(z) \gamma^{2}$ and primes denote the derivatives with respect to $\gamma$. Similar transformations hold also for $\bar{j}^{A}$.

In the following we consider the correlation functions of the primary fields $\Phi_{j}$. For later use, we expand $\Phi_{j}$ in terms of $e^{-2 \phi}$ :

$$
\Phi_{j}=\Phi_{j}^{\mathrm{f}}\left(1+\mathcal{O}\left(e^{-2 \phi}\right)\right)
$$

where

$$
\Phi_{j}^{\mathrm{f}}(z, x)=|x-\gamma|^{4 j} e^{2 j \phi}
$$

The correlator of $\Phi_{j}$ is written as

$$
\begin{aligned}
& \left\langle\prod_{a=1}^{N} \Phi_{j_{a}}\left(z_{a}, x_{a}\right)\right\rangle \\
& \quad=\int \mathcal{D} \phi \mathcal{D}\left(e^{\phi} \gamma\right) \mathcal{D}\left(e^{\phi} \bar{\gamma}\right) \mathcal{D}\left(e^{-\phi} \beta\right) \mathcal{D}\left(e^{-\phi} \bar{\beta}\right) \exp [-S] \prod_{a=1}^{N} \Phi_{j_{a}}^{\mathrm{f}}\left(z_{a}, x_{a}\right)\left(1+\mathcal{O}\left(e^{-2 \phi}\right)\right) .
\end{aligned}
$$

From the index theorem for the fields $(\beta, \gamma)$ with spin $(1,0)$, the zero-mode part of the measure is

$$
d \phi_{0} d \gamma_{0} d \bar{\gamma}_{0} d^{g} \beta_{0} d^{g} \bar{\beta}_{0} e^{2(1-g) \phi_{0}}
$$

where $\phi_{0}, \gamma_{0}, \ldots$ are the zero-modes of the respective fields and $g$ is the genus of the worldsheet. In this paper, we focus on the case $g=0$, in which the above expression becomes $d \phi_{0} d^{2} \gamma_{0} e^{2 \phi_{0}}$.

An important point in our argument is that one can first perform the integration over $\phi_{0}$. The $\phi_{0}$ integral in $(3.7)$ reads

$$
\begin{aligned}
& \int d \phi_{0} e^{2\left(\Sigma_{a} j_{a}+1\right) \phi_{0}} \exp \left[e^{-2 \phi_{0}} \int \frac{d^{2} w}{k \pi} \beta \bar{\beta} e^{-2 \phi_{q}}\right] \cdot\left(1+\mathcal{O}\left(e^{-2 \phi}\right)\right) \\
= & \frac{1}{2} \Gamma\left(-\sum_{a} j_{a}-1\right)\left[-\int \frac{d^{2} w}{k \pi} \beta \bar{\beta} e^{-2 \phi_{q}}\right]^{\sum_{a} j_{a}+1} \cdot\left(1+\mathcal{O}\left(e^{-2 \phi_{q}}\right)\right),
\end{aligned}
$$


where $\phi_{q}$ denotes the non-zero mode of $\phi$ and $\mathcal{O}\left(e^{-2 \phi_{q}}\right)$ represents the contributions from the higher order terms in $e^{-2 \phi_{q}}$. Since the interaction term $S_{\text {int }}$ appears only in the above form, the remaining functional integration reduces to that with respect to the free action $S_{0}$.

Next we perform the functional integration over the non-zero modes. The functional determinant coming from the integration over $\beta \gamma$ and $\bar{\beta} \bar{\gamma}$ gives a shift of the kinetic term of $\phi$ :

$$
\operatorname{det}^{-1}\left(e^{-\phi_{q}} \partial e^{2 \phi_{q}} \bar{\partial} e^{-\phi_{q}}\right)=\exp \left[\int d^{2} z\left(\frac{2}{\pi} \partial \phi \bar{\partial} \phi+\frac{\phi_{q}}{4 \pi} \sqrt{g} R\right)\right] .
$$

The resultant expression for the correlation function of the primaries is then

$$
\begin{aligned}
& \left\langle\prod_{a=1}^{N} \Phi_{j_{a}}\left(z_{a}, x_{a}\right)\right\rangle \\
& \quad=\frac{1}{2} \Gamma\left(-\sum_{a} j_{a}-1\right) \int d \gamma_{0} d \bar{\gamma}_{0}\left\langle\prod_{a=1}^{N} \Phi_{j_{a}}^{\mathrm{f}}\left(z_{a}, x_{a}\right)\left(1+\mathcal{O}\left(e^{-2 \phi}\right)\right) S_{\mathrm{int}}^{\Sigma_{a} j_{a}+1}\right\rangle_{\phi_{0}=0, \gamma_{0}, \bar{\gamma}_{0}}^{\mathrm{f}}
\end{aligned}
$$

where the bracket $\langle A\rangle_{\phi_{0}=0, \gamma_{0}, \bar{\gamma}_{0}}^{\mathrm{f}}$ represents the Wick contraction of $A$ using

$$
\begin{aligned}
& \phi(z)=\phi_{0}+\phi_{q}(z), \quad\left\langle\phi_{q}(z) \phi_{q}(w)\right\rangle_{\phi_{0}, \gamma_{0}, \gamma_{0}}^{\mathrm{f}}=-b^{2} \ln |z-w| \\
& \gamma(z)=\gamma_{0}+\gamma_{q}(z), \quad\left\langle\beta(z) \gamma_{q}(w)\right\rangle_{\phi_{0}, \gamma_{0}, \gamma_{0}}^{\mathrm{f}}=(z-w)^{-1}
\end{aligned}
$$

with $b^{-2}=k-2$.

Furthermore, similarly to the discussions in [19, 15], one can show that the $\mathcal{O}\left(e^{-2 \phi}\right)$ terms disappear after the renormalization because of the self-contraction of $e^{\phi\left(z_{a}\right)} \mathrm{s}$ (at least when the calculation can be carried out). Hence we arrive at the expression

$$
\left\langle\prod_{a=1}^{N} \Phi_{j_{a}}\left(z_{a}, x_{a}\right)\right\rangle=\frac{1}{2} \Gamma\left(-\sum_{a} j_{a}-1\right) \int d \gamma_{0} d \bar{\gamma}_{0}\left\langle\prod_{a=1}^{N} \Phi_{j_{a}}^{\mathrm{f}}\left(z_{a}, x_{a}\right) S_{\mathrm{int}} \Sigma_{a} j_{a}+1\right\rangle_{\phi_{0}=0, \gamma_{0}, \bar{\gamma}_{0}}^{\mathrm{f}} .
$$

Since it turns out that $\Phi_{j}^{\mathrm{f}}$ correspond to the primaries in a free theory, the right-hand side is nothing but a correlation function in a free theory with $\gamma_{0}$-integral. $S_{\text {int }}$ plays the role of the screening operator.

An anomaly term as in (3.9) may also be obtained by changing the measure (3.2) to

$$
\mathcal{D} \phi \mathcal{D} \gamma \mathcal{D} \bar{\gamma} \mathcal{D} \beta \mathcal{D} \bar{\beta}
$$

Such a term and $S_{0}$ then add up to

$$
S_{\text {free }}=\frac{1}{\pi} \int d^{2} z\left[(k-2) \partial \phi \bar{\partial} \phi-\frac{\phi}{4} \sqrt{g} R-\beta \bar{\partial} \gamma-\bar{\beta} \partial \bar{\gamma}\right] .
$$

The same $\phi_{0}$ dependence as in (3.8) comes from the term $\phi \sqrt{g} R$ and a similar calculation in the above is possible. However, in this approach, the full $S L(2)$ symmetry (3.4) appears

\footnotetext{
1 We omit the terms which arise because of the background metric.
} 
subtle: in the full interacting theory, it is difficult to evaluate the Jacobian from (3.2) to (3.13) and Jacobians of the type $\mathcal{D}\left(e^{\epsilon(\gamma)} \gamma\right) / \mathcal{D} \gamma$, which are needed to check the invariance under (3.4).2

Alternatively, the expression (3.12) can be obtained by starting with the free theory with the action $S_{\text {free }}$ and a perturbation term $S_{\text {int }}$. In this picture, all fields are free fields from the beginning and, for example, $\beta, \gamma(\bar{\beta}, \bar{\gamma})$ constitute a holomorphic (anti-holomorphic) bosonic ghost system. $\Phi_{j}^{\mathrm{f}}$ are the primary fields with respect to the standard free field $S L(2)$ currents:

$$
\hat{j}^{-}=\beta, \quad \hat{j}^{3}=\beta \gamma+b^{-2} \partial \phi, \quad \hat{j}^{+}=\beta \gamma^{2}+2 b^{-2} \gamma \partial \phi+k \partial \gamma .
$$

In fact, $\Phi_{j}^{\mathrm{f}}$ satisfy the OPEs (2.3) with $\hat{j}^{A}$ and have worldsheet conformal weight $h \equiv-b^{2} j(j+$ $1)$. One then finds that the interaction $S_{\text {int }}$ is made of a screening current $\beta \bar{\beta} e^{-2 \phi}$ which has no singular OPEs with $\hat{j}^{A}$ up to total derivatives.

Generic correlators in this case are defined as follows:

$$
\langle X[\phi, \beta, \gamma, \bar{\beta}, \bar{\gamma}]\rangle \equiv \int \mathcal{D} \phi \mathcal{D} \gamma \mathcal{D} \bar{\gamma} \mathcal{D} \beta \mathcal{D} \bar{\beta} \exp \left[-S_{\text {free }}\right] \cdot X \exp \left[-S_{\text {int }}\right]
$$

Now the $S L(2)$ currents $\hat{j}^{A}$ are associated to the following symmetry of the path-integration:

$$
\begin{array}{r}
\int \mathcal{D} \phi^{\prime} \mathcal{D} \gamma^{\prime} \mathcal{D} \bar{\gamma}^{\prime} \mathcal{D} \beta^{\prime} \mathcal{D} \bar{\beta}^{\prime} \exp \left[-S_{\text {free }}^{\prime}\right]=\int \mathcal{D} \phi \mathcal{D} \gamma \mathcal{D} \bar{\gamma} \mathcal{D} \beta \mathcal{D} \bar{\beta} \exp \left[-S_{\text {free }}\right], \\
\gamma^{\prime}=\gamma+\epsilon, \quad \beta^{\prime}=\beta-\epsilon^{\prime} \beta-b^{-2} \epsilon^{\prime \prime} \partial \phi+\frac{k}{2} \partial \epsilon^{\prime \prime}, \quad \phi^{\prime}=\phi-\frac{1}{2} \epsilon^{\prime},
\end{array}
$$

where $\epsilon$ is as given in (3.4). However, the above transformation leaves $S_{\text {int }}$ invariant only up to terms proportional to the equation of motion. Hence it is an on-shell or perturbative symmetry, but not the symmetry of the full theory based on the original action (2.1).

Integrating over non-zero modes in (3.16), we obtain

$$
\langle X\rangle=\int d \phi_{0} d \gamma_{0} d \bar{\gamma}_{0} d^{g} \beta_{0} d^{g} \bar{\beta}_{0} e^{2(1-g) \phi_{0}}\left\langle X \exp \left[-S_{\text {int }}\right]\right\rangle_{\phi_{0}, \gamma_{0}, \bar{\gamma}_{0}, \beta_{0}, \bar{\beta}_{0}}^{\mathrm{f}},
$$

where the bracket $\langle A\rangle_{\phi_{0}, \ldots}^{\mathrm{f}}$ represents the Wick contraction of $A$ as before. For correlators of primaries on a sphere, we actually obtain the same expression as in (3.12) by substituting $X=\prod_{a=1}^{N} \Phi_{j_{a}}^{\mathrm{f}}\left(z_{a}, x_{a}\right)$ and integrating over $\phi_{0}$.

Although we can obtain the same expressions for correlators, the underlying symmetry (3.17) is different from that of the full interacting theory as discussed above. Hence it is more appropriate to start from the full treatment if we try to analyze the original theory with the full symmetry (3.4) and regard the invariance of correlation functions as originating from the true symmetry of the Lagrangian.

\footnotetext{
2 Strictly speaking, one needs to check that the regularization in calculating (3.9) respects the $S L(2)$ symmetry. However, our results indicate that the procedure in the above actually respect it in total.

${ }^{3}$ Here, there is a subtlety again in evaluating Jacobians such as $\mathcal{D}\left(e^{\epsilon(\gamma)} \gamma\right) / \mathcal{D} \gamma$.
} 
The procedure leading to (3.12) may be an analog of the Liouville case discussed by Goulian and $\mathrm{Li}$ [35] (see also [36, 37, 38]). Since the $H_{3}^{+}$theory is a little more complicated than Liouville theory, we needed to take into account the renormalization in addition to the integral over $\phi_{0}$.

Using the free field prescription obtained in this way and, in particular, the formula (3.12), we would like to obtain the explicit forms of the correlators and analytically continue them in $j_{a}$ similarly to [39, 40]. Such a continuation may be justified along the same line as in the Liouville case [41, 42]. Indeed, we will see that our correlators are in complete agreement with the exact results obtained by other approaches [28, 29, 15].

For the expression (3.12) to make sense, $\sum_{a} j_{a}+1$ should be a non-negative integer, but the prefactor $\Gamma\left(-\sum_{a} j_{a}-1\right)$ is then divergent. Similar divergences appear also in Liouville theory. In that case, they arise inevitably if we define correlation functions as analytic functions of complex $j_{a}$ [39, 40]. The situation in our case seems similar and $\sum_{a} j_{a}+1 \in \mathbf{Z}_{\geq 0}$ may be interpreted as a kind of "mass-shell" condition according to the Liouville case 44, 40.

Also, the free field approach is usually taken to be valid as $\phi \rightarrow \infty$, namely, near the boundary of $A d S_{3}$, because the interaction term $S_{\text {int }}$ is vanishing there. The primaries $\Phi_{j}$

reduce to $\Phi_{j}^{\mathrm{f}}$ in that limit. However, an important consequence of our argument is that the free field approach is more powerful as long as we consider the correlation functions of the primaries.

Finally, we would like to comment on the relationship to [15]. Our new prescription differs from those in [19 and 15] in which correlators are defined by taking projections onto the $S L(2, \mathbf{C})$-invariant part by hand and introducing delta-functionals such as $\delta^{2}\left(e^{2 \phi\left(z_{0}\right)} \gamma\left(z_{0}\right)\right)$. Because of such functionals, $N$-point functions are seemingly represented by $(N+1)$-point functions in an ordinary sense. On the other hand, in our formula, an $S L(2, \mathbf{C})$-invariant result (in the sense of space-time) is obtained by the integration over the zero-modes of $\phi, \gamma, \bar{\gamma}$, and the calculation is very much simplified. One can confirm such simplifications in the following examples of the two- and three-point functions.

\subsection{Two-Point Function}

For the two-point function, the formula (3.12) and the integration over $\gamma_{0}, \bar{\gamma}_{0}$ yield

$$
\begin{aligned}
& \left\langle\Phi_{j_{1}}\left(z_{1}, x_{1}\right) \Phi_{j_{2}}\left(z_{2}, x_{2}\right)\right\rangle \\
& =\frac{\pi}{2} \Gamma\left(-j_{1}-j_{2}-1\right) \Delta\left(2 j_{1}+1\right) \Delta\left(2 j_{2}+1\right) \Delta\left(-j_{1}-j_{2}\right) \Gamma\left(j_{1}+j_{2}+2\right)^{-2}\left|x_{12}\right|^{2 j_{1}+2 j_{2}} \\
& \quad \cdot\left\langle\left|\gamma_{12}\right|^{2 j_{1}+2 j_{2}+2} e^{2 j_{1} \phi\left(z_{1}\right)} e^{2 j_{2} \phi\left(z_{2}\right)}\left[-S_{\text {int }}\right]^{j_{1}+j_{2}+1}\right\rangle_{\phi_{0}=\gamma_{0}=\bar{\gamma}_{0}=0}^{\mathrm{f}}
\end{aligned}
$$

Here $\gamma_{12}=\gamma\left(z_{1}\right)-\gamma\left(z_{2}\right), x_{12}=x_{1}-x_{2}$ and we have introduced $\Delta(x) \equiv \Gamma(x) / \Gamma(1-x)$. The integration over $\gamma_{0}$ can be carried out using the formula

$$
\int d^{2} x|x|^{4 j_{1}}|1-x|^{4 j_{2}}=\pi \Delta\left(2 j_{1}+1\right) \Delta\left(2 j_{2}+1\right) \Delta\left(-2 j_{1}-2 j_{2}-1\right),
$$


and then we expanded a binomial $\left|\gamma_{12}-x_{12}\right|^{4 j_{1}+4 j_{2}+2}$ and picked up the relevant term. The remaining free CFT correlator is given by a Dotsenko-Fateev integral [44]:

$$
\begin{aligned}
& \frac{1}{\Gamma\left(j_{1}+j_{2}+2\right)^{2}}\left\langle\left|\gamma_{12}\right|^{2 j_{1}+2 j_{2}+2} e^{2 j_{1} \phi\left(z_{1}\right)} e^{2 j_{2} \phi\left(z_{2}\right)}\left[\int \frac{d^{2} w}{k \pi} \beta \bar{\beta} e^{-2 \phi}(w)\right]^{j_{1}+j_{2}+1}\right\rangle_{\phi_{0}=\gamma_{0}=\bar{\gamma}_{0}=0}^{\mathrm{f}} \\
& =\left|z_{12}\right|^{2 b^{2} j_{1}\left(j_{1}+1\right)+2 b^{2} j_{2}\left(j_{2}+1\right)} K\left(j_{1}-\frac{1}{2 b^{2}}, j_{2}-\frac{1}{2 b^{2}}, 0\right),
\end{aligned}
$$

where

$$
\begin{aligned}
K\left(\alpha_{1}, \alpha_{2}, \alpha_{3}\right) & \equiv \int \prod_{i=1}^{n} \frac{d^{2} y_{i}}{k \pi}\left|y_{i}\right|^{4 b^{2} \alpha_{1}}\left|1-y_{i}\right|^{4 b^{2} \alpha_{2}} \prod_{i<j}\left|y_{i}-y_{j}\right|^{-4 b^{2}} \\
& =\frac{\left[k^{-1} b^{-2 b^{2}} \Delta\left(b^{2}\right)\right]^{n} \Upsilon[b] \Upsilon\left[-2 \alpha_{1} b\right] \Upsilon\left[-2 \alpha_{2} b\right] \Upsilon\left[-2 \alpha_{3} b\right]}{\Gamma(n+1) \Upsilon\left[-\left(\sum \alpha_{i}+1\right) b\right] \Upsilon\left[-\alpha_{12} b\right] \Upsilon\left[-\alpha_{23} b\right] \Upsilon\left[-\alpha_{31} b\right]}
\end{aligned}
$$

Here the integral is expressed using the $\Upsilon$-function. The definition and some basic properties of $\Upsilon(x)$ are found, e.g., in the appendix of [15].

In calculating further, note that $K\left(\alpha_{i}\right)$ becomes delta-functional in the limit $\alpha_{3} \rightarrow 0$ with the support on the zeroes of the denominator. Analyzing in a similar way as in [29, 15], we see that the relevant zeroes of the denominator are at $j_{1}+j_{2}+1=0$ and at $j_{1}=j_{2}$. Thus we obtain

$$
\begin{aligned}
\left\langle\Phi_{j_{1}}\left(z_{1}, x_{1}\right) \Phi_{j_{2}}\left(z_{2}, x_{2}\right)\right\rangle & \\
& =\left|z_{12}\right|^{4 b^{2} j_{1}\left(j_{1}+1\right)}\left[A\left(j_{1}\right) \delta^{2}\left(x_{12}\right) i \delta\left(j_{1}+j_{2}+1\right)+B\left(j_{1}\right)\left|x_{12}\right|^{4 j_{1}} i \delta\left(j_{1}-j_{2}\right)\right], \\
A(j) & =-\frac{\pi^{3}}{(2 j+1)^{2}}, \\
B(j) & =b^{2} \pi^{2}\left[k^{-1} \Delta\left(b^{2}\right)\right]^{2 j+1} \Delta\left[-b^{2}(2 j+1)\right] .
\end{aligned}
$$

Comparing this with the result of [15], we see that they agree precisely up to an overall numerical factor. We also find an agreement with [28, 29] by appropriate changes of normalizations of the primaries.

\subsection{Three-Point Function}

In calculating the three-point function, one has to make use of the $S L(2)$ symmetry (2.3), and extract the $x_{a}$-dependence in the following way:

$$
\begin{aligned}
\left\langle\prod_{a=1}^{3} \Phi_{j_{a}}\left(z_{a}, x_{a}\right)\right\rangle & =\prod_{a<b}\left|x_{a b}\right|^{2 j_{a b}} D\left(j_{a}, z_{a}\right) \\
D\left(j_{a}, z_{a}\right) & =\left.\prod_{a<b}\left|x_{a b}\right|^{-2 j_{a b}}\left\langle\prod_{a=1}^{3} \Phi_{j_{a}}\left(z_{a}, x_{a}\right)\right\rangle\right|_{x_{1,2,3}=0,1, \infty}
\end{aligned}
$$


where we have used the notation $j_{12}=j_{1}+j_{2}-j_{3}$, etc. The coefficient $D\left(j_{a}, z_{a}\right)$ can then be calculated using (3.12). After separating the $z_{a}$-dependence by means of the worldsheet $S L(2, \mathbf{C})$ invariance, we find that the remaining part is again described by a Dotsenko-Fateev integral:

$$
\begin{aligned}
D\left(j_{a}, z_{a}\right)= & \prod_{a<b}\left|z_{a b}\right|^{-2 h_{a b}} \cdot \frac{\pi}{2} \Gamma\left(-\Sigma j_{a}-1\right) \Delta\left(2 j_{1}+1\right) \Delta\left(2 j_{2}+1\right) \Delta\left(-j_{12}\right) \\
& \cdot K\left(j_{1}-\frac{1}{2 b^{2}}, j_{2}-\frac{1}{2 b^{2}}, j_{3}\right) .
\end{aligned}
$$

Here we have used $h_{12}=h_{1}+h_{2}-h_{3}$, etc. Summarizing, the three-point function is given by

$$
\begin{gathered}
\left\langle\prod_{a=1}^{3} \Phi_{j_{a}}\left(z_{a}, x_{a}\right)\right\rangle=D\left(j_{a}\right) \prod_{a<b}\left|z_{a b}\right|^{-2 h_{a b}}\left|x_{a b}\right|^{2 j_{a b}} \\
D\left(j_{a}\right)=\frac{b^{2} \pi}{2} \frac{\left[k^{-1} b^{-2 b^{2}} \Delta\left(b^{2}\right)\right]^{\Sigma j_{a}+1} \Upsilon[b] \Upsilon\left[-2 j_{1} b\right] \Upsilon\left[-2 j_{2} b\right] \Upsilon\left[-2 j_{3} b\right]}{\Upsilon\left[-\left(\Sigma j_{a}+1\right) b\right] \Upsilon\left[-j_{12} b\right] \Upsilon\left[-j_{13} b\right] \Upsilon\left[-j_{23} b\right]} .
\end{gathered}
$$

This is again in precise agreement with the known results.

Before concluding this section, we would like to note that our two- and three-point functions are consistent with the following symmetry of primary fields:

$$
\begin{aligned}
\Phi_{j}(z, x) & =R(j) \int d^{2} y|x-y|^{4 j} \Phi_{-j-1}(z, y), \\
R(j) & =-\frac{(2 j+1)^{2} b^{2}}{\pi} \Delta\left[-(2 j+1) b^{2}\right]\left[k^{-1} \Delta\left(b^{2}\right)\right]^{2 j+1} .
\end{aligned}
$$

This is understood as a non-trivial check that the procedure in subsection 3.1 respected the $S L(2)$ symmetry.

It is straightforward to write down an integral formula for $N$-point functions of primary fields. We will give the explicit expression in the $N=4$ case in the next section.

\section{Solving Knizhnik-Zamolodchikov EQUATION}

To see that our prescription works also for four-point functions, we would like to obtain some of them from a different approach: by solving the Knizhnik-Zamolodchikov(KZ) equation. We will find that some solutions obtained in this way are also calculated correctly and easily from our prescription in section 3.

For generic correlators of primaries, the $\mathrm{KZ}$ equation is given by

$$
\begin{gathered}
{\left[\partial_{z_{a}}-b^{2} \sum_{b(\neq a)} z_{a b}^{-1} \mathcal{L}_{a b}\right]\left\langle\prod_{c} \Phi_{j_{c}}\left(z_{c}, x_{c}\right)\right\rangle=0,} \\
\mathcal{L}_{a b}=x_{a b}^{2} \partial_{x_{a}} \partial_{x_{b}}-2 x_{a b}\left(j_{a} \partial_{x_{b}}-j_{b} \partial_{x_{a}}\right)-2 j_{a} j_{b} .
\end{gathered}
$$


In the case of four-point functions, the worldsheet and space-time $S L(2, \mathbf{C})$ invariances determine their form up to an arbitrary function of cross ratios:

$$
\begin{gathered}
\left\langle\prod_{a=1}^{4} \Phi_{j_{a}}\left(z_{a}, x_{a}\right)\right\rangle=\prod_{a<b}^{4}\left|z_{a b}\right|^{2 b^{2} \mu_{a b}}\left|x_{a b}\right|^{2 \lambda_{a b}} F(z, x), \\
z \equiv \frac{z_{41} z_{23}}{z_{43} z_{21}}, \quad x \equiv \frac{x_{41} x_{23}}{x_{43} x_{21}} .
\end{gathered}
$$

If we choose $\lambda_{a b}$ and $\mu_{a b}$ in the following way,

$$
\begin{array}{lll}
\lambda_{12}=j_{1}+j_{2}-j_{3}+j_{4}, & \mu_{12}=\Delta_{1}+\Delta_{2}-\Delta_{3}+\Delta_{4}+2 j_{1} j_{4}+2 j_{2} j_{4}, \\
\lambda_{13}=j_{1}-j_{2}+j_{3}-j_{4}, & \mu_{13}=\Delta_{1}-\Delta_{2}+\Delta_{3}-\Delta_{4}-2 j_{2} j_{4}, \\
\lambda_{14}=0, & \mu_{14}=-2 j_{1} j_{4}, \\
\lambda_{23}=-j_{1}+j_{2}+j_{3}-j_{4}, & \mu_{23}=-\Delta_{1}+\Delta_{2}+\Delta_{3}-\Delta_{4}-2 j_{1} j_{4}, \\
\lambda_{24}=0, & \mu_{24}=-2 j_{2} j_{4}, \\
\lambda_{34}=2 j_{4}, & \mu_{34}=2 \Delta_{4}+2 j_{1} j_{4}+2 j_{2} j_{4},
\end{array}
$$

with $\Delta_{a}=j_{a}\left(j_{a}+1\right)$, the $\mathrm{KZ}$ equation for $F(z, x)$ becomes

$$
\begin{gathered}
0=\left[\frac{1}{b^{2}} \frac{\partial}{\partial z}+\frac{x P_{0}}{z}+\frac{(1-x) P_{1}}{z-1}\right] F \\
P_{i}=x(1-x) \partial_{x}^{2}+\left[\gamma_{i}-(1+\alpha+\beta) x\right] \partial_{x}-\alpha \beta \\
\alpha=-2 j_{4}, \quad \beta=-j_{1}-j_{2}+j_{3}-j_{4}, \quad \gamma_{0}=-2 j_{1}-2 j_{4}, \quad \gamma_{1}=1-j_{1}+j_{2}+j_{3}-j_{4} .
\end{gathered}
$$

We see that $P_{i}$ in the above are nothing but the hypergeometric differentials. Hence it is expected that, under certain conditions on $j_{a}$, the solution can be written down explicitly using hypergeometric functions. In the following we will give some examples in which the $\mathrm{KZ}$ equation is solved rather easily.

\section{$4.1 \quad \sum_{a} j_{a}=-1$}

The simplest example is the case $\sum_{a} j_{a}=-1$. Since $\gamma_{0}=\gamma_{1}$ and the two hypergeometric differentials coincide in this case, one can find a solution with $F(z, x)$ independent of $z$. The four-point function is therefore given by

$$
\begin{aligned}
\left\langle\prod_{a=1}^{4} \Phi_{j_{a}}\left(z_{a}, x_{a}\right)\right\rangle= & \left|x_{12}\right|^{2\left(j_{1}+j_{2}-j_{3}+j_{4}\right)}\left|x_{13}\right|^{2\left(j_{1}-j_{2}+j_{3}-j_{4}\right)}\left|x_{23}\right|^{2\left(-j_{1}+j_{2}+j_{3}-j_{4}\right)}\left|x_{34}\right|^{4 j_{4}} \\
& \cdot \prod_{a<b}^{4}\left|z_{a b}\right|^{-4 b^{2} j_{a} j_{b}} \cdot f(x)
\end{aligned}
$$


where $f(x)$ satisfies the hypergeometric differential equation $P_{0} f(x)=0$. Recalling that $f(x)$ must be real and single-valued if $j_{a}$ are all real, one obtains

$$
\begin{aligned}
f(x) & =C \int d^{2} t|t|^{2 \beta-2}|1-t|^{2 \gamma_{0}-2 \beta-2}|1-t x|^{-2 \alpha} \\
& =C \int d^{2} t|t|^{4 j_{3}}|1-t|^{4 j_{2}}|1-t x|^{4 j_{4}},
\end{aligned}
$$

where $C$ is a constant. Actually this $f(x)$ is understood as a naive "square", i.e., monodromyinvariant combination, of the hypergeometric function represented as a contour integral.

Making a change of the integration variable from $t$ to $x_{0}$ defined by

$$
t=\frac{x_{03} x_{21}}{x_{01} x_{23}}
$$

the solution can be recast in a form which is manifestly symmetric in four vertices:

$$
\left\langle\prod_{a=1}^{4} \Phi_{j_{a}}\left(z_{a}, x_{a}\right)\right\rangle=C\left(j_{a}\right) \prod_{a<b}^{4}\left|z_{a b}\right|^{-4 b^{2} j_{a} j_{b}} \cdot \int d^{2} x_{0} \prod_{a}\left|x_{0 a}\right|^{4 j_{a}} .
$$

\section{2 $\quad \sum_{a} j_{a}=0$}

The next simplest is the case $\sum_{a} j_{a}=0$ or $\gamma_{1}=\gamma_{0}+1$. In this case the two hypergeometric functions $F\left(\alpha, \beta, \gamma_{i} ; x\right)$ have a special property: the differentials $x P_{0}$ and $(1-x) P_{1}$ are linearly realized on them. This is due to the following recursion relation of hypergeometric functions:

$$
\begin{aligned}
\gamma(1-x) \frac{\partial}{\partial x} F(\alpha, \beta, \gamma ; x) & =(\gamma-\alpha)(\gamma-\beta) F(\alpha, \beta, \gamma+1 ; x)+\gamma(\alpha+\beta-\gamma) F(\alpha, \beta, \gamma ; x) \\
x \frac{\partial}{\partial x} F(\alpha, \beta, \gamma+1 ; x) & =\gamma\{F(\alpha, \beta, \gamma ; x)-F(\alpha, \beta, \gamma+1 ; x)\}
\end{aligned}
$$

Therefore the solution to the KZ equation can be found as a linear combination of the two hypergeometric functions $F\left(\alpha, \beta, \gamma_{i} ; x\right)$,

$$
F(z, x) \sim(z-1) g_{0}(z) F\left(\alpha, \beta, \gamma_{0} ; x\right)+z g_{1}(z) F\left(\alpha, \beta, \gamma_{1} ; x\right)
$$

or of another solutions to $P_{i} f_{i}(x)=0$. Here " $"$ means that we do not care about the dependence on $\bar{z}$ and $\bar{x}$ for the time being.

Putting the above ansatz into the KZ equation, we obtain

$$
\begin{aligned}
\frac{\partial}{b^{2} \partial z}\left[(z-1) g_{0}\right] & =\gamma_{0} g_{1}-\left(\alpha+\beta-\gamma_{0}\right) g_{0} \\
\frac{\partial}{b^{2} \partial z}\left[z g_{1}\right] & =-\gamma_{0} g_{1}-\frac{\left(\gamma_{0}-\alpha\right)\left(\gamma_{0}-\beta\right)}{\gamma_{0}} g_{0} .
\end{aligned}
$$

The previous recursion relation is utilized here again, and we obtain a solution to the KZ equation which has the following form:

$$
\begin{array}{r}
F(z, x) \sim \gamma_{0}\left(1+b^{2} \gamma_{0}\right)(1-z) F\left(1+b^{2} \alpha, 1+b^{2} \beta, 1+b^{2} \gamma_{0} ; z\right) F\left(\alpha, \beta, \gamma_{0} ; x\right) \\
+b^{2}\left(\gamma_{0}-\alpha\right)\left(\gamma_{0}-\beta\right) z F\left(1+b^{2} \alpha, 1+b^{2} \beta, 2+b^{2} \gamma_{0} ; z\right) F\left(\alpha, \beta, \gamma_{0}+1 ; x\right)
\end{array}
$$


Since there are two solutions to each hypergeometric differential equation, we have three other independent solutions to the KZ equation which have a similar form.

Now we rewrite the above solution using the contour integral expression for the hypergeometric function, so that the permutation symmetry among four vertices becomes manifest. Writing $F(z, x)$ as

$$
\begin{aligned}
F(z, x) & =\gamma_{0}\left(1+b^{2} \gamma_{0}\right)(1-z) G_{0}(z) F_{0}(x)+b^{2}\left(\gamma_{0}-\alpha\right)\left(\gamma_{0}-\beta\right) z G_{1}(z) F_{1}(x), \\
G_{0}(z) & =\frac{1}{1-e^{2 \pi i\left(b^{2} \beta+1\right)}} \frac{\Gamma\left(b^{2} \gamma_{0}+1\right)}{\Gamma\left(b^{2} \beta+1\right) \Gamma\left(b^{2}\left(\gamma_{0}-\beta\right)\right)} \oint d t t^{b^{2} \beta}(1-t)^{b^{2}\left(\gamma_{0}-\beta\right)-1}(1-t x)^{-b^{2} \alpha-1}, \\
G_{1}(z) & =\frac{1}{1-e^{2 \pi i\left(b^{2} \beta+1\right)}} \frac{\Gamma\left(b^{2} \gamma_{0}+2\right)}{\Gamma\left(b^{2} \beta+1\right) \Gamma\left(b^{2}\left(\gamma_{0}-\beta\right)+1\right)} \oint d t t^{b^{2} \beta}(1-t)^{b^{2}\left(\gamma_{0}-\beta\right)}(1-t x)^{-b^{2} \alpha-1}, \\
F_{0}(x) & =\frac{1}{1-e^{2 \pi i \beta}} \frac{\Gamma\left(\gamma_{0}\right)}{\Gamma(\beta) \Gamma\left(\gamma_{0}-\beta\right)} \oint d s s^{\beta-1}(1-s)^{\gamma_{0}-\beta-1}(1-s x)^{-\alpha}, \\
F_{1}(x) & =\frac{1}{1-e^{2 \pi i \beta}} \frac{\Gamma\left(\gamma_{0}+1\right)}{\Gamma(\beta) \Gamma\left(\gamma_{0}-\beta+1\right)} \oint d s s^{\beta-1}(1-s)^{\gamma_{0}-\beta}(1-s x)^{-\alpha}
\end{aligned}
$$

and making the change of variables from $s, t$ to $x_{0}, z_{0}$ which are defined by

$$
s=\frac{x_{03} x_{21}}{x_{01} x_{23}}, \quad t=\frac{z_{03} z_{21}}{z_{01} z_{23}},
$$

we can rewrite $F(z, x)$ in the following form:

$$
\prod_{a<b}^{4} z_{a b}{ }^{2} \mu_{a b} x_{a b}{ }^{\lambda_{a b}} F(z, x) \sim \prod_{a<b}^{4} z_{a b}{ }^{-2 b^{2} j_{a} j_{b}} \oint d z_{0} d x_{0} \prod_{a} z_{0 a}^{2 b^{2} j_{a}} x_{0 a}^{2 j_{a}} \cdot \sum_{a} \frac{j_{a}}{z_{0 a} x_{0 a}}
$$

The above expression is obviously symmetric in four vertices, and by taking its naive square we obtain the four-point function:

$$
\left\langle\prod_{a=1}^{4} \Phi_{j_{a}}\left(z_{a}, x_{a}\right)\right\rangle=C\left(j_{a}\right) \prod_{a<b}\left|z_{a b}\right|^{-4 b^{2} j_{a} j_{b}} \int d^{2} z_{0} d^{2} x_{0} \prod_{a}\left|z_{0 a}\right|^{4 b^{2} j_{a}}\left|x_{0 a}\right|^{4 j_{a}} \cdot\left|\sum_{a} \frac{j_{a}}{z_{0 a} x_{0 a}}\right|^{2} .
$$

The other solutions to the KZ equation can be obtained by starting from (4.12) with $\alpha$ and $\beta$ in $F_{0,1}(x)$ exchanged each other. Proceeding in a similar way we obtain

$$
\begin{aligned}
z_{a b}^{b^{2} \mu_{a b}} x_{a b}^{\lambda_{a b}} F(z, x) \sim z_{a b}^{-2 b^{2} j_{a j} j_{b}} \oint d z_{0} d x_{0} \prod_{a} z_{0 a}^{2 b^{2} j_{a}} x_{0 a}^{2 j_{a}} \\
\cdot \cdot\left\{\left(x_{01}^{-1}-x_{04}^{-1}\right)\left(z_{02}^{-1}-z_{04}^{-1}\right)-\left(x_{02}^{-1}-x_{04}^{-1}\right)\left(z_{01}^{-1}-z_{04}^{-1}\right)\right\} .
\end{aligned}
$$

By exchanging four vertices we obtain different solutions, but the number of independent solutions is three. By summing over their squares with an appropriate weight, it might be possible to construct a quantity satisfying consistency conditions such as the single-valuedness, symmetry between four vertices, etc. However, it cannot reduce to the known three-point function of the $\mathrm{H}_{3}^{+}$WZW model in the limit $j_{4} \rightarrow 0$ as one can see by a comparison with 
the free field result in section 3. Note that, since our KZ equation is a partial differential equation, there exist large degrees of freedom of the solutions.

\subsection{Comparison with Free Field Approach}

By simple calculation we see that (3.12) with $N=4$ gives the solutions to the $\mathrm{KZ}$ equation, (4.8) and (4.14), up to factors. In this identification, the integration variables $x_{0}$ and $z_{0}$ in (4.8) and (4.14) correspond to the zero-mode of $\gamma$ and the position of the screening current $\beta \bar{\beta} e^{-2 \phi}$, respectively.

We can write down the integral formula for more generic four-point functions. If $\sum_{a} j_{a}+1$ is a non-negative integer, the four-point function is expressed in terms of a free field correlator with $\sum_{a} j_{a}+1$ insertions of $S_{\mathrm{int}}$ :

$$
\begin{aligned}
& \left\langle\prod_{a=1}^{4} \Phi_{j_{a}}\left(z_{a}, x_{a}\right)\right\rangle=\frac{1}{2} \Gamma\left(-\Sigma j_{a}-1\right)(-k \pi)^{-\Sigma j_{a}-1} \prod_{a<b}\left|x_{a b}\right|^{2 \lambda_{a b}}\left|z_{a b}\right|^{2 b^{2} \mu_{a b}}|f(z, x)|^{2} \\
& f(z, x)=\frac{\Gamma\left(j_{1}-j_{2}+j_{3}-j_{4}+1\right)}{\Gamma\left(-2 j_{2}\right) \Gamma\left(-2 j_{4}\right)} \int d \gamma_{0} \cdot \gamma_{0}^{2 j_{1}} \\
& \quad \cdot \int_{0}^{1} d s d t \delta(s+t-1) s^{-2 j_{2}-1} t^{-2 j_{4}-1}\left(\gamma_{0}-s-t x\right)^{-j_{1}+j_{2}-j_{3}+j_{4}-1} \\
& \quad \cdot \int_{i=1}^{\Sigma j_{a}+1} d y_{i}\left(\frac{s}{y_{i}-1}+\frac{t z}{y_{i}-z}\right) y_{i}^{2 b^{2} j_{1}-1}\left(y_{i}-1\right)^{2 b^{2} j_{2}}\left(y_{i}-z\right)^{2 b^{2} j_{4}} \prod_{i<j} y_{i j}^{-2 b^{2}} .
\end{aligned}
$$

As a remark, we note that our new formula with an additional integration over the zeromode of $\gamma$ is related to the well-studied case $j_{1}+j_{2}-j_{3}+j_{4} \in \mathbf{Z}_{\geq 0}$ in the literature. In this case, using the free field realization of the $S L(2)$ current, the solutions to the $\mathrm{KZ}$ equation for $F(z, x)$ have been given by the free field correlators with the insertion of $j_{1}+j_{2}-j_{3}+j_{4}$ screening charges [23, 25, 27]. Note that, when $j_{1}+j_{2}-j_{3}+j_{4} \in \mathbf{Z}_{\geq 0}$, the KZ equation can be solved rather easily, because $\beta$ in (4.5) is a negative integer and the hypergeometric function then reduces to a polynomial of $x$. Those solutions and ours may be connected through the relation (3.24) between $\Phi_{j_{3}}$ and $\Phi_{-j_{3}-1}$. For example, the polynomials of $x$ in the former are converted to the hypergeometric functions in the latter by the $y$-integral in (3.24).

\section{Conclusions}

In this paper, we have argued that the calculation of the correlators in the $\mathrm{H}_{3}^{+} \mathrm{WZW}$ model is reduced to that of a free theory. The point is the integration over the zero-mode of $\phi$ and the renormalization.

Since the free field picture used in the calculation has been deduced from the full interacting theory, the results are expected to be exact. Indeed, we have found a precise agreement with the known exact results for the two- and three-point functions. Moreover, our prescription allows us to calculate higher point correlation functions including numerical factors, at 
least under some conditions. We have actually seen that the four-point functions which are obtained by solving the KZ equation can also be reproduced easily in our formalism.

In comparing the solutions to the KZ equation with our free field results, we identify one of the integration variables in the former with the zero-mode of $\gamma$ in the latter. This means that we have to deal with the zero-modes of the fields appropriately in calculating correlators even if we use the free field picture.

Admittedly, we have not yet succeeded in extracting the results directly relevant to the string theory on $A d S_{3}$. However, we believe that our argument here made a step toward a better understanding. Thus, let us discuss some possible directions. First, one advantage of using the free field picture may be that we can construct various vertices easily. For example, we need some free field realization of the current algebra to construct vertices for spectralflowed representations [10]. The correlators containing such vertices may also be calculated in our approach.

Second, it is interesting to study the singular behavior of the four-point function when $z$ approaches $x$ in $F(z, x)$. In some recent work, such a singularity is identified with the singularity involving a "long string" with a unit winding number [46]. To investigate the four-point functions further, it may be useful to use the relationship between the four-point function in the $S L(2)$ case and the five-point function in the minimal models 445, 23, 25]

Regarding the AdS/CFT correspondence, the Virasoro central charge of the space-time CFT might be calculable from our approach. It would be interesting to obtain its precise value and consider why it has to be quantized. Though the quantization is considered to be a non-perturbative effect as has been discussed in [6], we might be able to explain it from our approach.

Finally, we would like to note the similarity between our formalism and that for Liouville theory. In particular, in Liouville theory or the two-dimensional string theory, the $N$-point correlators can be obtained when the moduli are integrated out [43]. In our case, such correlators correspond to the correlators of the "space-time" CFT. Our discussion in this paper suggests that the correlators of the space-time CFT may be analyzed using the techniques developed for Liouville theory.

\section{ACKNOWLEDGMENTS}

We would like to thank N. Ishibashi for useful discussions and correspondences. We are also grateful to the organizers of "Summer Institute 2000" at Yamanashi, Japan, where a part of this work has been carried out. The works of K.H. and K.O. were supported in part by JSPS Research Fellowships for Young Scientists. The work of Y.S. was supported in part by Grant-in-Aid for Scientific Research on Priority Area 707 from the Ministry of Education, Science and Culture in Japan. 


\section{REFERENCES}

[1] J. Maldacena, "The large N limit of superconformal field theories and supergravity," Adv. Theor. Math. Phys. 2, 231 (1998), hep-th/9711200.

[2] S.S. Gubser, I.R. Klebanov and A.M. Polyakov, "Gauge theory correlators from noncritical string theory," Phys. Lett. B428, 105 (1998), hep-th/9802109.

[3] E. Witten, "Anti-de Sitter space and holography," Adv. Theor. Math. Phys. 2, 253 (1998), hep-th/9802150.

[4] A. Giveon, D. Kutasov and N. Seiberg, "Comments on string theory on $A d S_{3}$," Adv. Theor. Math. Phys. 2, 733 (1998), hep-th/9806194.

[5] J. de Boer, H. Ooguri, H. Robins and J. Tannenhauser, "String theory on $A d S_{3}$," JHEP 9812, 026 (1998), hep-th/9812046.

[6] D. Kutasov and N. Seiberg, "More comments on string theory on $A d S_{3}$," JHEP 9904, 008 (1999), hep-th/9903219.

[7] I. Bars, C. Deliduman and D. Minic, "String theory on $A d S_{3}$ revisited," hep-th/9907087.

[8] P.M. Petropoulos, "String theory on $A d S_{3}$ : some open questions," hep-th/9908189.

[9] G. Giribet and C. Nunez, "Interacting strings on $A d S_{3}$, , JHEP 9911, 031 (1999), hep-th/9909149.

[10] J. Maldacena and H. Ooguri, "Strings in $A d S_{3}$ and $S L(2, \mathbf{R})$ WZW model: I," hep-th/0001053.

[11] A. Kato and Y. Satoh, "Modular invariance of string theory on $A d S_{3}$," Phys. Lett. B486, 306 (2000), hep-th/0001063.

[12] A.L. Larsen and N. Sanchez, "Quantum coherent string states in $A d S_{3}$ and $S L(2, \mathbf{R})$ WZWN model," Phys. Rev. D62, 046003 (2000), hep-th/0001180.

[13] I. Pesando, "Some remarks on the free fields realization of the bosonic string on $A d S_{3}$," hep-th/0003036.

[14] Y. Hikida, K. Hosomichi and Y. Sugawara, "String theory on $A d S_{3}$ as discrete lightcone Liouville theory," Nucl. Phys. B589, 134 (2000), hep-th/0005065.

[15] N. Ishibashi, K. Okuyama and Y. Satoh, "Path integral approach to string theory on $A d S_{3}, "$ Nucl. Phys. B588, 149 (2000), hep-th/0005152.

[16] J. Maldacena, H. Ooguri and J. Son, "Strings in $A d S_{3}$ and the $S L(2, \mathbf{R}) W Z W$ model. II: Euclidean black hole," hep-th/0005183.

[17] G. Giribet and C. Nunez, "Aspects of the free field description of string theory on $A d S_{3}$, , JHEP 0006, 033 (2000), hep-th/0006070.

[18] Z. Haba, "Correlation functions of sigma fields with values in a hyperbolic space," Int. J. Mod. Phys. A4, 267 (1989). 
[19] K. Gawędzki, "Quadrature of conformal field theories", Nucl. Phys. B328 (1989) 733; "Non-compact WZW conformal field theories", NATO ASI: Cargese 1991: 0247-274, hep-th/9110076.

[20] D. Bernard and G. Felder, "Fock representations and BRST cohomology in SL(2) current algebra," Commun. Math. Phys. 127, 145 (1990).

[21] V.S. Dotsenko, "The free field representation of The SU(2) conformal field theory," Nucl. Phys. B338, 747 (1990);

"Solving the $S U(2)$ conformal field theory with the Wakimoto free field representation," Nucl. Phys. B358, 547 (1991).

[22] A. Gerasimov, A. Morozov, M. Olshanetsky, A. Marshakov and S. Shatashvili, "WessZumino-Witten model as a theory of free fields," Int. J. Mod. Phys. A5, 2495 (1990).

[23] P. Furlan, A.C. Ganchev, R. Paunov and V.B. Petkova, "Reduction of the rational spin sl $(2, C)$ WZNW conformal theory," Phys. Lett. B267, 63 (1991);

"Solutions of the Knizhnik-Zamolodchikov equation with rational isospins and the reduction to the minimal models," Nucl. Phys. B394, 665 (1993), hep-th/9201080.

[24] O. Andreev, "Operator algebra of the $S L(2)$ conformal field theories," Phys. Lett. B363, 166 (1995), hep-th/9504082.

[25] J.L. Petersen, J. Rasmussen and M. Yu, "Conformal blocks for admissible representations in SL(2) current algebra," Nucl. Phys. B457, 309 (1995), hep-th/9504127;

"Hamiltonian reduction of SL(2) theories at the level of correlators," Nucl. Phys. B457, 343 (1995), hep-th/9506180.

[26] O. Andreev, "On affine Lie superalgebras, $A d S_{3} / C F T$ correspondence and world-sheets for world-sheets," Nucl. Phys. B552, 169 (1999), hep-th/9901118;

"Unitary representations of some infinite dimensional Lie algebras motivated by string theory on $A d S_{3}$, , Nucl. Phys. B561, 413 (1999) hep-th/9905002.

[27] K. Saraikin, "Conformal blocks and correlators in WZNW model. I: Genus zero," hep-th/9912042.

[28] J. Teschner, "On structure constants and fusion rules in the $S L(2, \mathbf{C}) / S U(2) W Z N W$ model," Nucl. Phys. B546, 390 (1999), hep-th/9712256;

"The mini-superspace limit of the $S L(2, \mathbf{C}) / S U(2) W Z N W$ model," Nucl. Phys. B546, 369 (1999), hep-th/9712258.

[29] J. Teschner, "Operator product expansion and factorization in the $\mathrm{H}_{3}^{+}$WZNW model," Nucl. Phys. B571, 555 (2000), hep-th/9906215.

[30] I.I. Kogan, A. Lewis and O.A. Soloviev, "Gauge Dressing of 2D Field Theories," Int. J. Mod. Phys. A12, 2425 (1997), hep-th/9607048;

"Knizhnik-Zamolodchikov-type equations for gauged WZNW models," Int. J. Mod. Phys. A13, 1345 (1998), hep-th/9703028. 
[31] M.J. Bhaseen, I.I. Kogan, O.A. Soloviev, N. Taniguchi and A. Tsvelik, "Towards a Field Theory of the Plateau Transition," Nucl. Phys. B580, 688 (2000), cond-mat/9912060.

[32] I.I. Kogan and A.M. Tsvelik, "Logarithmic Operators in the Theory of Plateau Transition," Mod. Phys. Lett. A15, 931 (2000), hep-th/9912143.

[33] A. Nichols and Sanjay, "Logarithmic operators in the $S L(2, \mathbf{R})$ WZNW model," hep-th/0007007.

[34] A. Lewis, "Logarithmic CFT on the Boundary and the World-Sheet," hep-th/0009096.

[35] M. Goulian and M. Li, "Correlation functions in Liouville theory," Phys. Rev. Lett. 66, 2051 (1991).

[36] E. Braaten, T. Curtright, G. Ghandour and C. Thorn, "Nonperturbative weak coupling analysis of the Liouville quantum field theory," Phys. Rev. Lett. 51, 19 (1983).

[37] A. Gupta, S. P. Trivedi and M. B. Wise, "Random Surfaces In Conformal Gauge," Nucl. Phys. B340, 475 (1990).

[38] M. Bershadsky and D. Kutasov, "Comment on gauged WZW theory," Phys. Lett. B266, 345 (1991).

[39] H. Dorn and H.J. Otto, "Two and three point functions in Liouville theory," Nucl. Phys. B429, 375 (1994), hep-th/9403141.

[40] A.B. Zamolodchikov and Al.B. Zamolodchikov, "Structure constants and conformal bootstrap in Liouville field theory", Nucl. Phys. B477, 577 (1996), hep-th/9506136.

[41] K. Aoki and E. D'Hoker, "On the Liouville approach to correlation functions for 2-D quantum gravity," Mod. Phys. Lett. A7, 235 (1992), hep-th/9109024.

[42] J. Gervais, "Gravity - matter couplings from Liouville theory," Nucl. Phys. B391, 287 (1993), hep-th/9205034.

[43] P. Di Francesco and D. Kutasov, "World sheet and space-time physics in twodimensional (super)string theory," Nucl. Phys. B375, 119 (1992), hep-th/9109005.

[44] V.S. Dotsenko and V.A. Fateev, "Conformal algebra and multipoint correlation functions in 2D statistical models," Nucl. Phys. B240, 312 (1984);

"Four point correlation functions and the operator algebra in the two-dimensional conformal invariant theories with the central charge $c<1$," Nucl. Phys. B251, 691 (1985).

[45] A.B. Zamolodchikov and V.A. Fateev, "Operator algebra and correlation functions in the two-dimensional $S U(2) \times S U(2)$ chiral Wess-Zumino model," Sov. J. Nucl. Phys. 43, 657 (1986).

[46] H. Ooguri, talk at Strings 2000, http://feynman.physics.lsa.umich.edu/cgi-bin/s2ktalk.cgi?ooguri 